\title{
Performances of a linear type magnetic flux pump for compensating a little decremented persistent current of HTS magnets
}

\author{
$\operatorname{AUTHOR}(S):$ \\ Chung, Y; Muta, I; Hoshino, T; Nakamura, T
}

\section{CITATION:}

Chung, Y ... [et al]. Performances of a linear type magnetic flux pump for compensating a little decremented persistent current of HTS magnets. IEEE TRANSACTIONS ON APPLIED SUPERCONDUCTIVITY 2004, 14(2): 1723-1726

\section{ISSUE DATE:}

2004-06

URL:

http://hdl.handle.net/2433/39955

\section{RIGHT:}

(c)2004 IEEE. Personal use of this material is permitted. However, permission to reprint/republish this material for advertising or promotional purposes or for creating new collective works for resale or redistribution to servers or lists, or to reuse any copyrighted component of this work in other works must be obtained from the IEEE. 


\title{
Performances of a Linear Type Magnetic Flux Pump for Compensating a Little Decremented Persistent Current of HTS Magnets
}

\author{
Yoondo Chung, Itsuya Muta, Tsutomu Hoshino, and Taketsune Nakamura
}

\begin{abstract}
This paper describes the characteristics of a linear type magnetic flux pump recently developed. The linear type flux pump has been fabricated to apply to compensate decremented persistent current of HTS applications such as NMR and MRI system. The linear type magnetic flux pump mainly consists of 3-phase AC armature coil, DC bias coil and superconducting $\mathrm{Nb}$ foil. In the preliminary experiment, the flux pump can charge the pumping-up current in the load coil of $1.3 \mathrm{mH}$ during 1000 seconds under the DC bias current of $10 \mathrm{~A}$ and 3-phase $\mathrm{AC}$ of $8 \mathrm{~A}, 60 \mathrm{~Hz}$. The magnitude of pumping current is $0.1 \mathrm{~mA} / \mathrm{s}$.
\end{abstract}

Index Terms-Linear type magnetic flux pump, Nb foil, persistent current compensation.

\section{INTRODUCTION}

$\mathbf{S}$ UPERCONDUCTING flux pump [1], [2] and superconducting rectifier have been proposed and developed for the past about fifty years. Most recently, the application for the superconducting power supply is very promising in high- $T_{c}$ superconducting (HTS) coils applications such as magnetic resonance image-CT (MRI-CT) and nuclear magnetic resonance spectrometer (NMR) used for life science fields. Compared with low- $T_{c}$ superconductors (LTS), since HTS coils have a low $n$-index, HTS coils could not keep the persistent current constant substantially [3], [4]. The linear type magnetic flux pump makes less vibrations and electric noises than the early-developed flux pump [5]-[7]. Consequently, a static linear type magnetic flux pump would be thought to be most suitable to compensate the decremented persistent current. Presently, the system of the flux pump has been performed for R\&D on current compensation source. In this experiment we observed the pumping-up current in the load coil to measure small quantities of pumping current.

We determined the ramping up rates of pumping current in the load coil under the DC bias current of $10 \mathrm{~A}$ and the 3-phase AC current of 8 A during 1000 seconds. The magnitude of pumping current is about $0.1 \mathrm{~mA} / \mathrm{s}$. The main purpose of this study is to obtain the design and manufacturing techniques for the compensator of decremented persistent current in the HTS magnet system.

Manuscript received October 21, 2003. This work was supported by Grant-in-Aid for exploratory research (no. 14655107) of JSPS and the 21st Century COE Program (no. 14213201) in Japan.

The authors are with the Electrical Engineering Department, Kyoto University, Kyoto 615-8510, Japan (e-mail: yoondo@asl.kuee.kyoto-u.ac.jp; muta@kuee.kyoto-u.ac.jp; hosinio@kuee.kyoto-u.ac.jp; tk_naka@kuee.kyotou.ac.jp).

Digital Object Identifier 10.1109/TASC.2004.831054

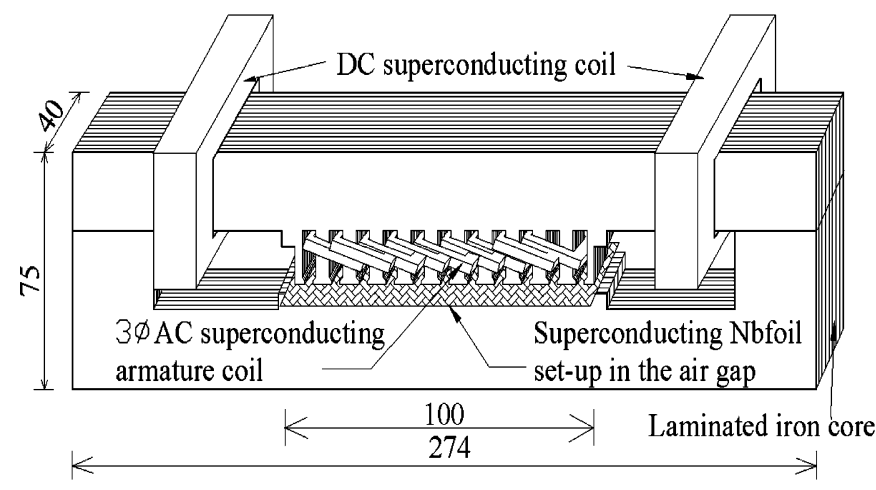

Fig. 1. Schematic diagram of the linear type magnetic flux pump.

\section{BASIC STRUCTURE AND CONCEPT}

\section{A. The Structure of the Linear Type Flux Pump}

The linear type flux pump is mainly composed of four components as follows:

1) Laminated linear core,

2) DC coil,

3) 3-phase AC coil,

4) Superconducting $\mathrm{Nb}$-foil.

The linear core of the flux pump was laminated to reduce eddy-current loss. NbTi conductors for DC are used as magnet coils; including a load coil of the flux pump. NbTi conductors for AC coils are used for 3-phase AC armature coils. A sheet of superconducting $\mathrm{Nb}$ foil, $20 \mu \mathrm{m}$ thick, $60 \mathrm{~mm}$ wide, 120 $\mathrm{mm}$ long, is set-up in the air gap of $3 \mathrm{~mm}$. The cover for superconducting $\mathrm{Nb}$ foil, which is made of translucent aluminum nitride ceramic, is installed to fix the $\mathrm{Nb}$ foil with high thermal conductivity. The connection between the load coil and the $\mathrm{Nb}$ foil is done by spot welding method.

Table I provides parameters for DC coil, load coil and 3-phase AC coil.

\section{B. The Concept and Operation of the Flux Pump}

Fig. 2 shows the schematic plot about magnetic flux density distributions in the air gap. The wounded AC armature coil, 3-phase and 2-pole, produces the traveling magnetic field. On the other hand, the DC coil produces some bias magnetic field, which contributes to move up the magnetic field near the zero level of magnetic flux density because the value of critical magnetic flux density is near the zero level as shown in Fig. 2. The DC bias current in the linear type flux pump produces a constant bias component. The homopole traveling wave by the DC bias 
TABLE I

PARAMETERS OF COILS

\begin{tabular}{|c|c|}
\hline \multicolumn{2}{|c|}{ DC COIL } \\
\hline Wire & $\mathrm{NbTi} / \mathrm{Cu}(1 / 3.3)$ \\
\hline Wire diameter [mm] & 0.9 \\
\hline Turns / coil & 132 \\
\hline Length / coil [m] & 23 \\
\hline Inside dimension $\left[\mathrm{mm}^{2}\right]$ & $3 \times 4.6$ \\
\hline Outside dimension $\left[\mathrm{mm}^{2}\right]$ & $4.8 \times 6.2$ \\
\hline \multicolumn{2}{|c|}{ LOAD COIL } \\
\hline Wire & $\mathrm{NbTi} / \mathrm{Cu}(1 / 3.3)$ \\
\hline Wire diameter [mm] & 0.9 \\
\hline Turns / coil & 142 \\
\hline Length / coil [m] & 72 \\
\hline Inner diameter [mm] & $\phi 40$ \\
\hline Outer diameter [mm] & $\phi 80$ \\
\hline Inductance $[\mathrm{mH}]$ & 1.3 \\
\hline \multicolumn{2}{|c|}{ 3-PHASE AC COIL } \\
\hline Wire & $\mathrm{NbTi}$ \\
\hline$I_{c} @ 4.2 \mathrm{~K}, 50 \mathrm{~Hz}\left[\mathrm{~A}_{\text {peak }}\right]$ & 42 \\
\hline Wire diameter [mm] & 0.6 \\
\hline Turns / phase & 40 \\
\hline Total length / phase [m] & 25 \\
\hline
\end{tabular}

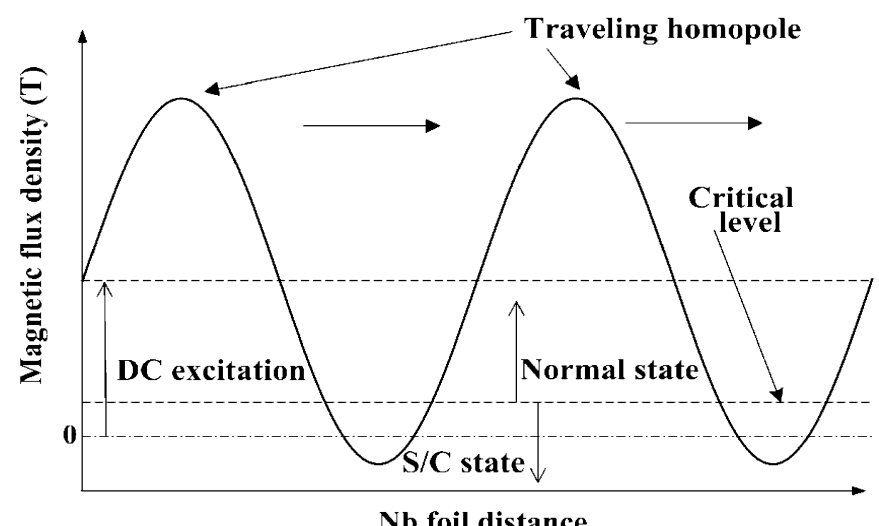

Fig. 2. Schematic plots for the superconducting $\mathrm{Nb}$ foil with $\mathrm{DC}$ and 3-phase AC excitations.

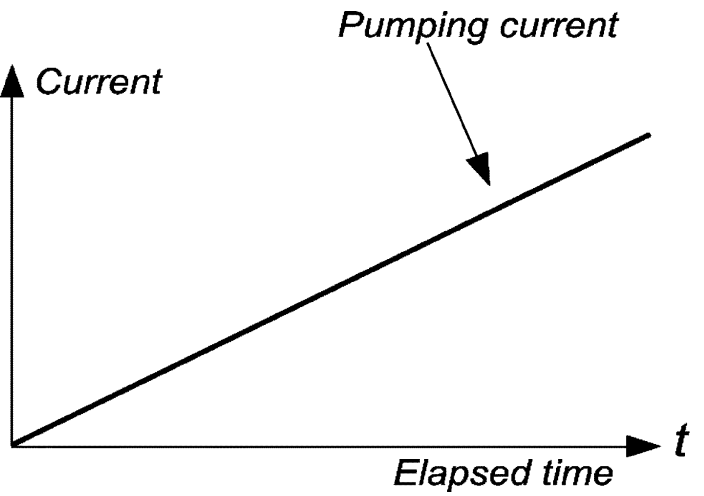

Fig. 3. Sequential diagram during the flux pump operation.

and the 3-phase AC current component produces S/N (superconducting to normal state) transitions into the $\mathrm{Nb}$ foil installed in the $3 \mathrm{~mm}$ air gap. Therefore, some magnetic fluxes can penetrate the $\mathrm{Nb}$ foil and the pumping-up current is accumulated in the superconductive circuit, which consists of the load coil connected to the $\mathrm{Nb}$ foil through superconducting wires. Although the homopolar traveling wave of Fig. 2 is an ideal sinusoidal wave, actual waveforms have many harmonics as shown in Figs. 6 and 7 later. Fig. 3 shows the ideal sequential diagram during the flux pumping operation.

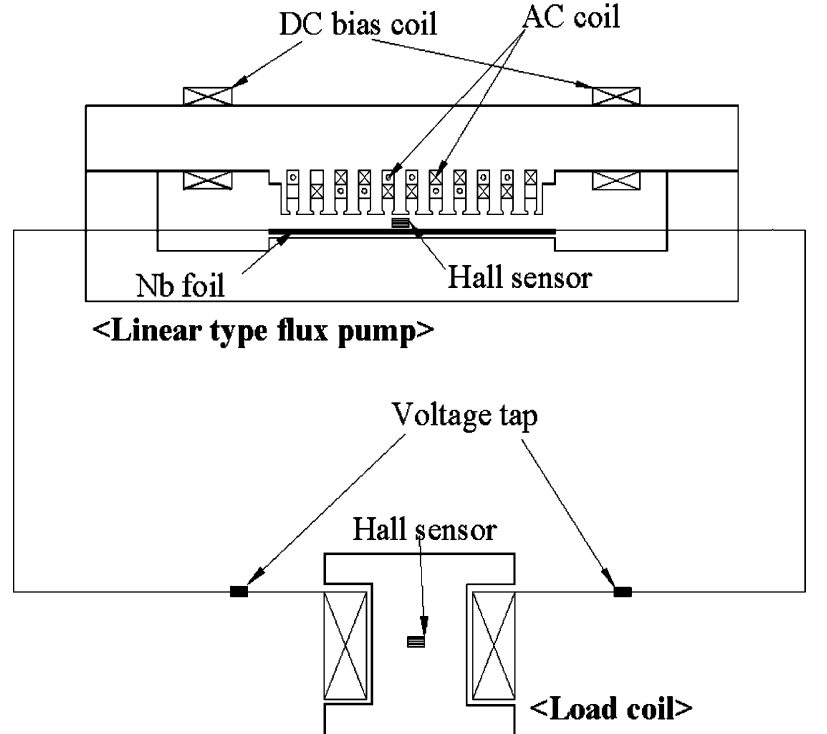

Fig. 4. Schematic diagram for the linear type flux pump system.

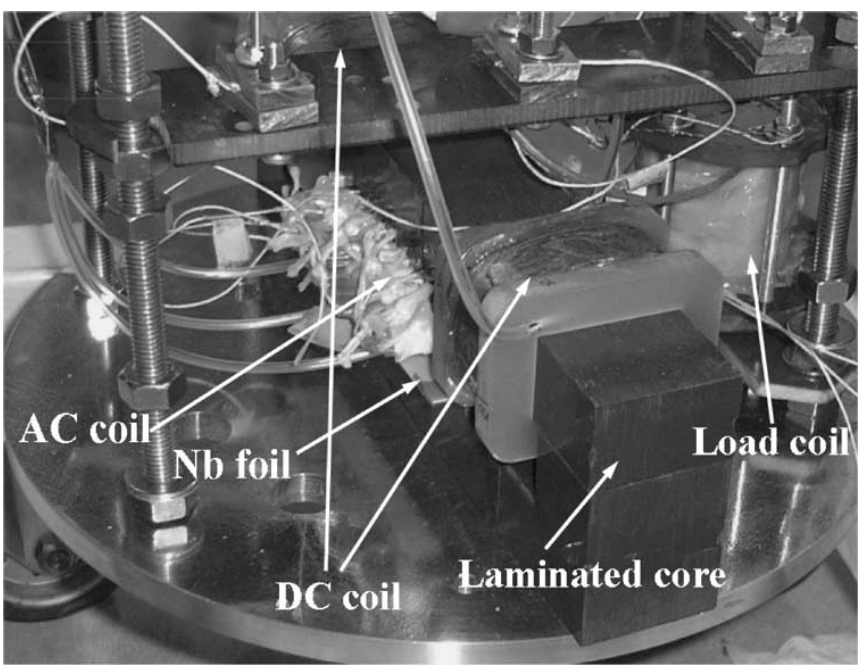

Fig. 5. Photograph of the linear type magnetic flux pump system.

\section{EXPERIMENTAL SETUP}

The connection diagram for experiment system circuit of the linear type magnetic flux pump system is shown in Fig. 4. In the flux pump system, 2 hall sensors are used; a transverse type cryogenic hall sensor to measure magnetic flux density at the center of the flux pump to determine the DC bias and AC excitations and an axial type hall sensor to calculate small increasing pumping current of the $\mathrm{NbTi}$ load coil. Also voltage tap installed both ends of the load coil. As the winding frame of the NbTi for DC coil is almost rectangular, we installed space bar at the edge part and on the surface of every layer. Inner side of the yoke of the linear type flux pump is installed with a slot liner to insulate between the laminated core and the AC coils. The DC coils and the load coil are wound on a GFRP bobbin and covered with stycast epoxy.

All signals are recorded and monitored through a data acquisition system at the same time. Shunt resistors are installed to measure the current value in the $\mathrm{DC}$ and 3-phase $\mathrm{AC}$ coils. All signals are passed through isolation amplifiers before being recorded in the recorder. Fig. 5 shows a photograph of the linear type magnetic flux pump system. 


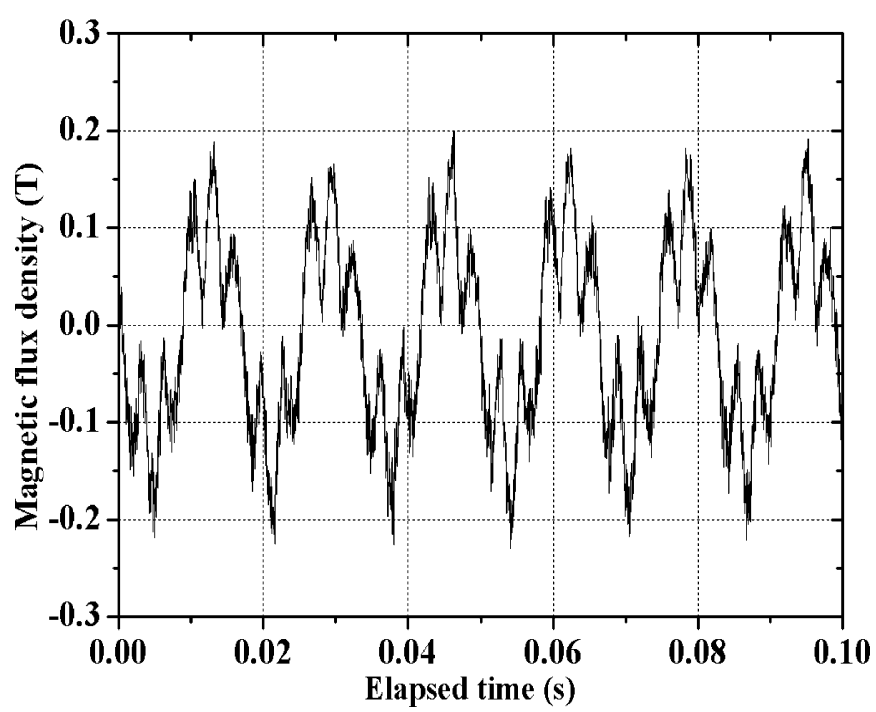

Fig. 6. Measured magnetic flux density at the linear type flux pump with 3-phase AC 8 A excitations at $60 \mathrm{~Hz}$.

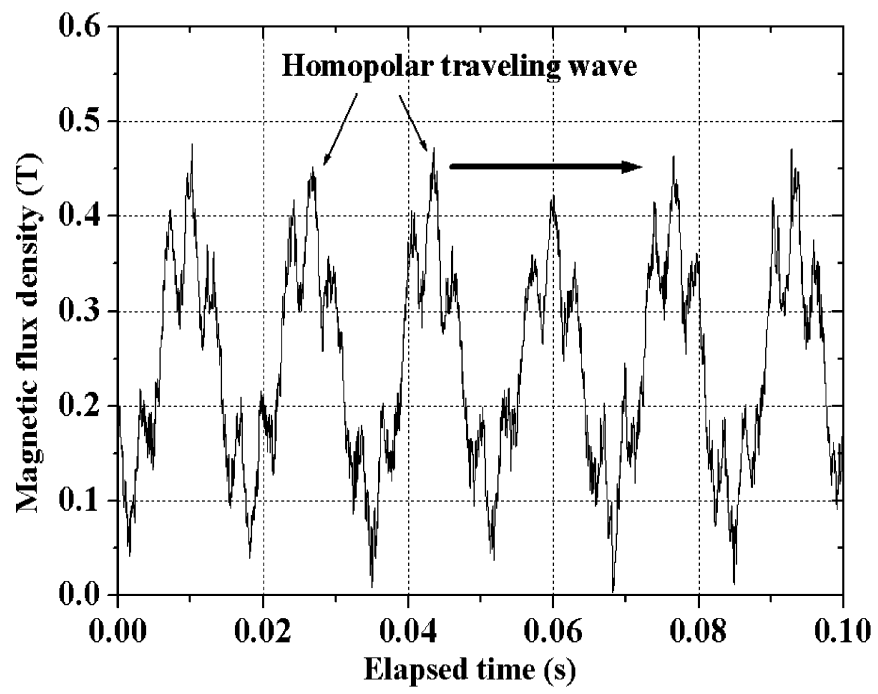

Fig. 7. Measured magnetic flux density at the linear type flux pump with DC bias of $10 \mathrm{~A}$ and 3-phase $\mathrm{AC}$ of $8 \mathrm{~A}$ excitations.

\section{RESUlTS AND DISCUSSION}

The pumping current and the magnet terminal voltage have been measured under the $\mathrm{DC}$ bias of $10 \mathrm{~A}$ and the 3-phase $\mathrm{AC}$ of $8 \mathrm{~A}$ at $60 \mathrm{~Hz}$. The examined magnetic flux density in the central air gap for the 3-phase $\mathrm{AC}$ of $8 \mathrm{~A}$ at $60 \mathrm{~Hz}$ is shown in Fig. 6. The maximum and minimum magnetic flux densities are about $0.2 \mathrm{~T}$ and $-0.2 \mathrm{~T}$, respectively. Fig. 7 shows the magnetic flux density in the central air gap for the DC bias of $10 \mathrm{~A}$ and the 3-phase $\mathrm{AC}$ of $8 \mathrm{~A}$ at $60 \mathrm{~Hz}$. The maximum and minimum magnetic flux densities are about $0.45 \mathrm{~T}$ and $0.05 \mathrm{~T}$. Fig. 8 shows the measured result of the load magnet voltage. Fig. 9 shows the measured pumping-up current during 1000 seconds. The pumping rate is about $0.1 \mathrm{~mA} / \mathrm{s}$. Through this preliminary test, we investigated that the pumping-up current at $60 \mathrm{~Hz}$ contained some fluctuations because the actual homopolar traveling wave form included some harmonics as shown in Figs. 6 and 7. Therefore, based on the measured result, it was investigated

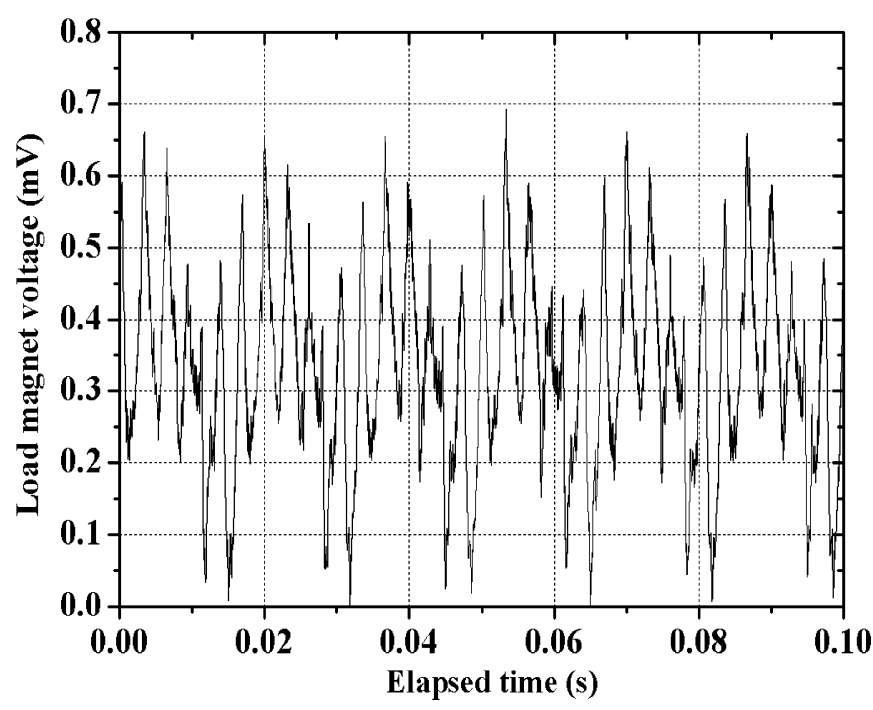

Fig. 8. Measured the load magnet voltage at $60 \mathrm{~Hz}$.

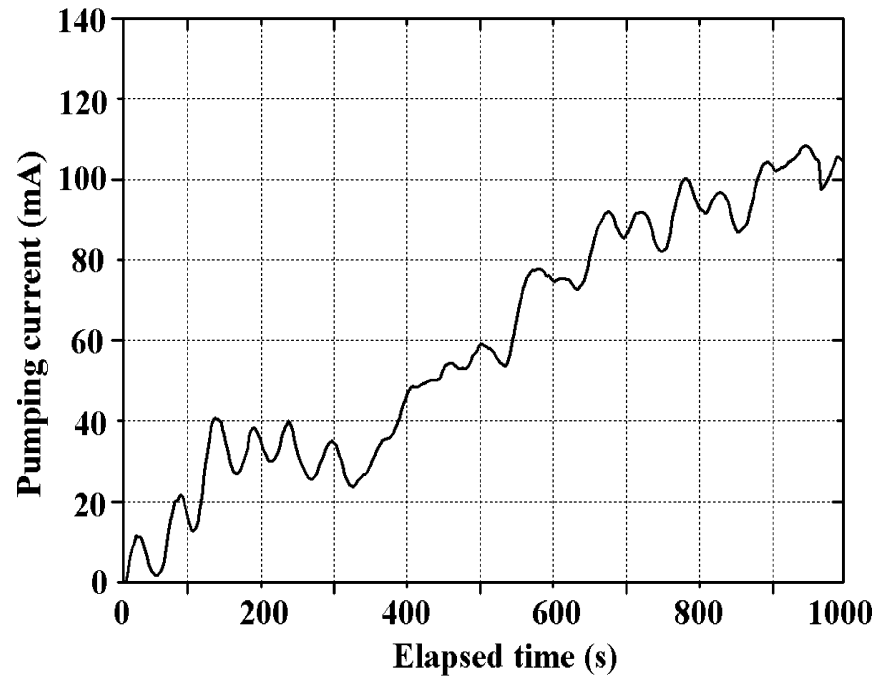

Fig. 9. Measured pumping-up current in the load magnet at $60 \mathrm{~Hz}$.

that the flux pump is required to reduce the harmonics of the homopolar traveling wave to obtain stable pumping-up current.

At the next stage, the flux pump will be tested under various frequencies of 3-phase $\mathrm{AC}$ current and magnitudes of $\mathrm{AC}$ current to control pumping-up current.

\section{CONCLUSION}

In this preliminary experiment, we have fabricated the linear type magnetic flux pump for compensating decremented persistent current of HTS magnets. It has been concluded that pumping rate reached about $0.1 \mathrm{~mA} / \mathrm{s}$ under the $\mathrm{DC}$ current of $10 \mathrm{~A}$ and the 3-phase AC current of $8 \mathrm{~A}$ at $60 \mathrm{~Hz}$. Based on the test, we obtained the design and manufacturing techniques for the persistent current compensator of HTS superconducting applications.

\section{REFERENCES}

[1] H. Tsukiji, T. Hoshino, and I. Muta, "Characteristics of exciting superconducting magnet by magnetic flux pump (in Japanese)," Trans. IEE Japan, vol. 115-D, no. 2, pp. 183-189, Feb. 1996. 
[2] H. Tsukiji, "Study on Brushless Superconducting Generator Excited by Magnetic Flux Pump," Ph.D. dissertation, Dec. 2000.

[3] Y. Iwasa, "Microampere flux pump for superconducting NMR magnets part 1; basic concept and microtesla flux measurement," Cryogenics, vol. 41, pp. 385-391, 2001.

[4] S. Jeong, "Experiment of flux pump for high temperature superconductor insert coils of NMR magnets," Journal of the Korea Institute of Applied Superconductivity and Cryogenics, vol. 3, no. 2, pp. 15-20, Nov. 2001.

[5] Y. Chung, I. Muta, T. Nakamura, and T. Hoshino, "Preparatory FEM analysis of a linear type magnetic flux pump for compensating a little decremented persistent current of HTS magnets," in 2002 Kansai-Section Joint Convention of Institutes of Electrical Eng. Japan, Nov. 2002, p. G123.
[6] - "Magnetic field distributions of a linear type magnetic flux pump to compensate a little decremented persistent current of HTS magnets for NMR using FEM," in 2003 National Convention IEE Japan, Mar. 2003, pp. 278-279.

[7] L. J. M. van de Klundert and H. H. J. ten Kate, "Fully superconducting rectifiers and flux pumps," Cryogenics, vol. 21, pp. 195-206, 1981. 